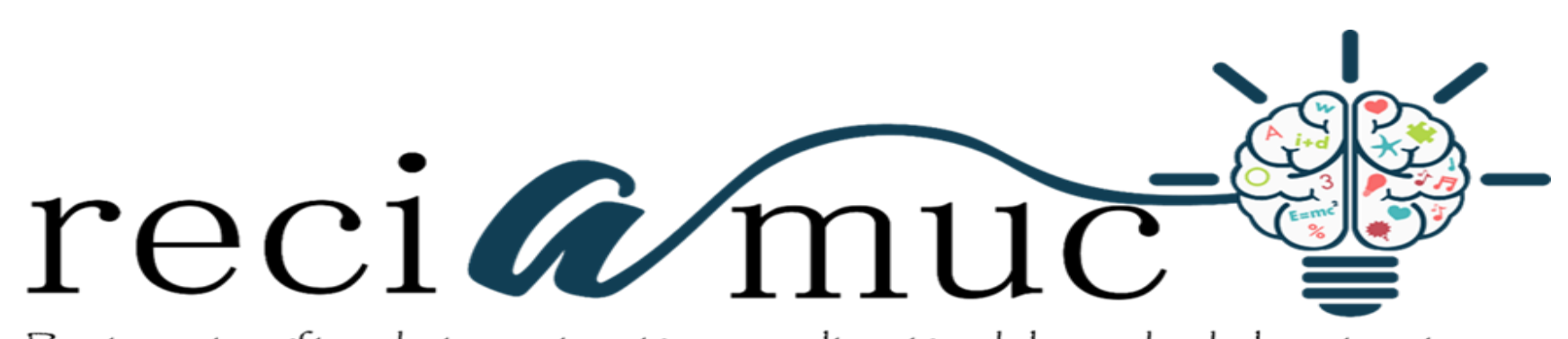

Revista cientifica de investigación actualización del mundo de las ciencias

Carolina Narcisa Chávez Ruíz a ; Luis Fernando Pascual García ${ }^{\text {b; }}$ Ximena Melissa

Gonzabay Jiménez ${ }^{\mathrm{c}}$; Diana Lilibeth Cruz Yoza ${ }^{\mathrm{d}}$

Injuria renal causada por AINEs

Kidney Injury caused by NSAIDs

Revista Científica de Investigación actualización del mundo de las Ciencias. Vol. 3 núm., 4, octubre: 2588-0748, 2019, pp. 3-21

DOI: $10.26820 /$ reciamuc/3.(4).octubre.2019.3-21

URL: http://reciamuc.com/index.php/RECIAMUC/article/view/385

Código UNESCO: 3205 Medicina Interna

Tipo de Investigación: Artículo de Revisión

(C) RECIAMUC; Editorial Saberes del Conocimiento, 2019

Recibido: $15 / 05 / 2019$

Aceptado: 07/08/2019

Publicado: 01/10/2019

Correspondencia: carolina_chavezr@ outlook.es

a. Médico; Investigador Independiente, Guayaquil, Ecuador; carolina_chavezr@outlook.es

b. Médico; Investigador Independiente, Guayaquil, Ecuador; luispascualgarcia@ hotmail.es

c. Médico; Investigador Independiente, Guayaquil, Ecuador; goxime311@ hotmail.com

d. Médico; Investigador Independiente, Guayaquil, Ecuador; diana_cruz1993@ hotmail.com 


\section{Injuria renal causada por AINEs}

Vol. 3, núm. 4., (2019)

Carolina Narcisa Chávez Ruíz; Luis Fernando Pascual García; Ximena Melissa Gonzabay Jiménez; Diana Lilibeth Cruz Yoza

\section{RESUMEN}

Elementalmente en medicina, la expresión Injuria Renal podría ser igualmente referente de falla, daño o insuficiencia a nivel renal. Los posibles efectos nefrotóxicos que los AINEs (Anti inflamatorios No Esteroideos); producen a nivel renal han sido tratados de explicar mediante la descripción de diferentes mecanismos, y precisamente uno de los principalmente descritos es la Injuria Renal Aguda (IRA/FRA), caso en el que se ha enfocado este trabajo investigativo. Se dice que los AINEs causan la insuficiencia renal aguda debido a que el propio efecto inhibitorio que éste ejerce sobre una función renal elemental, deja a dicho órgano en un desbalance que en definitiva conlleva a isquemia intrarenal, por ejemplo. Claro está que este no sería el único mecanismo que lo revela, de allí pues, surge el interés por investigar un poco más a detalle cuáles son las causas que en general explican el daño, la insuficiencia o el fallo a nivel renal de forma aguda en el ser humano adulto, de acuerdo a la literatura científico académica reciente. A tales fines, se adoptó un diseño documental secundario y una metodología de revisión sistemática, con la que se logró además exponer sobre la terminología básica asociada a dicha patología, síntomas y algunos aspectos fisiopatológicos, considerados necesarios para una comprensión del tema. Sé que concluye destacando lo importante que es; comprender que los AINEs son un grupo de fármacos que pueden producir múltiples afecciones a nivel renal; para el profesional de la salud, distinguir lo más adelantadamente posible a aquellos pacientes en los que se evidencie un riesgo elevado de ocurrencia de daño renal; y que tanto los pacientes como los profesionales de la salud, conozcan cada vez más a profundidad acerca de los efectos adversos que estos fármacos pueden causar, no solo a nivel renal sino también sobre otros órganos y sistemas.

Palabras Claves: Nefrotóxicos; Mecanismos; Inhibitorio; Desbalance; Daño. 


\title{
Injuria renal causada por AINEs
}

Vol. 3, núm. 4., (2019)

Carolina Narcisa Chávez Ruíz; Luis Fernando Pascual García; Ximena Melissa Gonzabay Jiménez; Diana Lilibeth Cruz Yoza

\begin{abstract}
Elementally in medicine, the expression Renal Injuria could also be a reference for failure, damage or insufficiency at the renal level. The possible nephrotoxic effects of NSAIDs (Non-Steroidal Anti-inflammatory); Produced at the renal level have been tried to explain by describing different mechanisms, and precisely one of the mainly described is Acute Renal Injuria (ARI / ARF), in which case this research work has been focused. It is said that NSAIDs cause acute renal failure because the inhibitory effect that it exerts on elementary renal function, leaves that organ in an imbalance that ultimately leads to intrarenal ischemia, for example. Of course, this would not be the only mechanism that reveals it, hence the interest arises to investigate a little more in detail what are the causes that generally explain the damage, insufficiency or failure at the renal level in an acute way. the adult human being, according to recent academic scientific literature. To this end, a secondary documentary design and a systematic review methodology were adopted, with which it was also possible to present about the basic terminology associated with said pathology, symptoms and some pathophysiological aspects, considered necessary for an understanding of the subject. I know it concludes by highlighting how important it is; understand that NSAIDs are a group of drugs that can produce multiple conditions at the renal level; for the health professional, distinguish as soon as possible those patients in whom there is a high risk of occurrence of renal damage; and that both patients and health professionals know more and more deeply about the adverse effects that these drugs can cause, not only at the renal level but also on other organs and systems.
\end{abstract}

Key words: Nephrotoxic; Mechanisms; Inhibitory; Imbalance; Damage. 


\section{Injuria renal causada por AINEs}

Vol. 3, núm. 4., (2019)

Carolina Narcisa Chávez Ruíz; Luis Fernando Pascual García; Ximena Melissa Gonzabay Jiménez; Diana Lilibeth Cruz Yoza

\section{Introducción.}

La injuria renal aguda (IRA) es una situación que mientras sea tratada adecuadamente y, sobre todo a tiempo, es reversible por completo en buena parte de los casos. En concreto se trata de pérdida brusca de la función renal. La disminución en el aporte de sangre al riñón, como causa más frecuente asociada a la IRA, se debe "a una mala irrigación, producto de hipotensión, deshidratación o alguna medicina que haya provocado algún tipo de alteración". (Hospital Aleman Asociación Civil, 2019)

Es por ello que, es vital que el profesional de la salud diagnostique esta patología lo antes posible.

Otra posible causa son los denominados nefrotóxicos, drogas que no cambian la irrigación del riñón, sino que producen un daño específico a sus células. Estas pueden ser antibióticos, analgésicos, antiinflamatorios, agentes utilizados en quimioterapia de cáncer, ciertos antihipertensivos y contraste radiológico (endovenoso).

Rechene, Fernández, \& Douthat (2018) dicen que, en la actualidad, se ha sugerido cambiar el uso del término IRA (relativo a insuficiencia renal aguda) por el de FRA, que significa fallo renal agudo, en vista de que se ha demostrado que pequeños cambios en la función renal, sin llegar a la insuficiencia, tienen gran relevancia en la morbimortalidad. Complementariamente informan que "el FRA representa un problema grave de salud actual, con un altísimo impacto y una elevada morbimortalidad asociada", a la par de un constante incremento anual en su incidencia a nivel mundial. Los autores alegan que, en base a diversos reportes, se conoce que la mortalidad 


\section{Injuria renal causada por AINEs}

Vol. 3, núm. 4., (2019)

Carolina Narcisa Chávez Ruíz; Luis Fernando Pascual García; Ximena Melissa Gonzabay Jiménez; Diana Lilibeth Cruz Yoza

intrahospitalaria representa un 37\% y la incidencia en unidades críticas se ubica entre el 5 y $57 \%$, rango destacadamente amplio debido a las diferentes definiciones usadas para el FRA, y a las distinciones en las características de los pacientes en cada institución y país. (Rechene, Fernández, \& Douthat; 2018; p. 171)

Los fármacos antiinflamatorios no esteroideos (AINEs), a menudo recetados en la práctica médica como analgésicos, antipiréticos y antiinflamatorios, son una de las clases de fármacos más utilizadas en todo el mundo. Estudios recientes señalan que los AINEs son los medicamentos más efectivos, por ejemplo, para tratar el dolor asociado con la crisis de cálculos renales, siendo superiores incluso a los opioides. Los mayores consumidores de este grupo de medicamentos son personas con dolor crónico, generalmente asociado con enfermedades reumatológicas, como artritis reumatoide, osteoartritis y otros trastornos musculoesqueléticos. La acción farmacológica de los AINEs depende de la dosis y el tiempo utilizado, lo que predispone la afectación de órganos específicos, siendo el segundo riñón más afectado. Por lo tanto, es uno de los medicamentos que, si se usa a largo plazo, aumenta la morbilidad, especialmente entre los ancianos, ya que usan varios medicamentos (antihipertensivos, antidepresivos, anticoagulantes) que pueden presentar interacciones. (Cavalcanti; et al.; 2018)

Varios estudios en sus resultados han dejado en evidencia la relación que al parecer existe entre el uso prolongado en altas dosis, combinado y sobretodo automedicado de Antiinflamatorios no esteroidales, con la nefropatía y la nefrotoxicidad, es por ello que el objetivo de este trabajo es exponer en relación al daño que el uso de AINEs en general causa al sistema renal o nefrológico y sus partes, así como también referir la terminología básica asociada a dicha patología, síntomas 


\section{Injuria renal causada por AINEs}

Vol. 3, núm. 4., (2019)

Carolina Narcisa Chávez Ruíz; Luis Fernando Pascual García; Ximena Melissa Gonzabay Jiménez; Diana Lilibeth Cruz Yoza

y algunos de sus aspectos fisiopatológicos disponibles como literatura cientificoacadémica más reciente en reconocidas bases de datos.

\section{Materiales y métodos}

Para esta investigación, se escoge un diseño documental secundario, contextualizado en una metodología de revisión sistemática

Esta investigación se enfoca en la búsqueda y revisión sistemática de literatura cientificoacadémica seleccionada, que por una parte, está disponible determinadas bases de datos, entre las que figuran: SciELO, Dialnet, MedlinePlus, Biblioteca Virtual de la Salud (BVS), Cochrane, entre otras; y por la otra, mediante el uso del material físicamente disponible en la biblioteca universitaria como en otras particulares, representando todo ello parte esencial del proceso investigativo y de comprensión que facilita la síntesis de la mejor evidencia disponible, y a su vez representando todo ello uno de los resultados esperados.

Principalmente se realiza una búsqueda aleatoria y consecutiva en las mencionadas bases de datos, usando las expresiones “injuria renal”, “injuria renal por AINE”, “insuficiencia renal aguda", "fallo renal agudo", lo que aproximadamente resultó en más de un centenar de miles de registros bibliográficos. Luego éstos se filtran en base a criterios de: idioma español, relevancia, correlación temática y fecha de publicación en los últimos siete años, sin descartar por tipo de material bibliográfico; es decir, se escogen títulos de artículos científicos, ensayos, revisiones sistemáticas, protocolos, editoriales, libros, boletines, folletos, tesis de grado, posgrado y 


\section{Injuria renal causada por AINEs}

Vol. 3, núm. 4., (2019)

Carolina Narcisa Chávez Ruíz; Luis Fernando Pascual García; Ximena Melissa Gonzabay Jiménez; Diana Lilibeth Cruz Yoza

doctorado, noticias científicas, entre otros documentos e información de interés científico y académico.

Seguidamente, se efectúa la selección y clasificación de la literatura científico académica físicamente disponible, aplicando, en términos generales, los criterios antes mencionados, siendo a partir de allí que el equipo investigador le da la correspondiente lectura crítica y análisis de toda esa evidencia científica, lo que resultó consecutiva y consensuadamente en el fundamento de las ideas y planteamientos aquí plasmados.

\section{Resultados}

Terminología básica

Antiinflamatorios. Son los medicamentos cuya función es la de detener o reducir la inflamación de una parte concreta del organismo, así como también para combatir la fiebre, como antitérmico (antipirético). Tales funciones están asociadas consecuentemente con una disminución del dolor asociado, produciendo también un efecto analgésico.

Existen dos grandes tipos de antiinflamatorios: los corticoesteroides, cuyo principio activo es la cortisona o sus derivados, y los llamados "AINEs", entre los que se encuentran estos principios activos bastante comunes: ibuprofeno, dexketoprofeno o naproxeno, diclofenaco, indometacina, entre otros. Aunque estos fármacos sean de uso cotidiano gracias a su mejor tolerabilidad, es muy importante seguir las indicaciones de los profesionales sanitarios, es decir, no se debe ser tan incauto al usarlo, debido a que un mal consumo o en exceso puede inducir el 


\section{Injuria renal causada por AINEs}

Vol. 3, núm. 4., (2019)

Carolina Narcisa Chávez Ruíz; Luis Fernando Pascual García; Ximena Melissa Gonzabay Jiménez; Diana Lilibeth Cruz Yoza

deterioro renal, complicaciones en el sistema digestivo y aumentar la presión arterial. (Kern Pharma, 2018)

Analgésicos. Sirven para reducir o aliviar el dolor (de cabeza, muscular o de articulación, entre otros) y la fiebre alta, porque al igual que los antiinflamatorios son antitérmicos. Entre los distintos tipos de analgésicos, están los más comunes son los que están en el grupo de los Antiinflamatorios No Esteroideos (AINEs), como el paracetamol. También están los opiáceos débiles, como los que llevan codeína o oxicodona; los opiáceos fuertes, como la morfina o el fentanilo; y por último los fármacos coadyuvantes, que son los que buscan aumentar la eficacia analgésica de los otros medicamentos. (Kern Pharma, 2018)

Nefropatías por AINEs. Especialmente los AINEs son causantes de nefropatías. El uso prolongado de este tipo de fármacos, como el ácido acetilsalicílico (aspirina) o ibuprofeno, inducen un daño dentro de las estructuras internas del riñón. (Enciclopedia Medica, 2017)

\section{Efecto nefrotóxico.}

Se entiende como la influencia "venenosa y dañina" para el riñón. Se asocia, particularmente en este caso, a la necrosis tubular aguda, la nefritis intersticial, la lesión glomerular y las alteraciones vasculares, que a su vez incluyen la microangiopatía trombótica, la aterosclerosis y la vasculitis. (Calderón, y otros, 2011) 


\section{Injuria renal causada por AINEs}

Vol. 3, núm. 4., (2019)

Carolina Narcisa Chávez Ruíz; Luis Fernando Pascual García; Ximena Melissa Gonzabay Jiménez; Diana Lilibeth Cruz Yoza

\section{Efecto vasoconstrictor.}

Es el estrechamiento (constricción) de vasos sanguíneos por parte de pequeños músculos en sus paredes. Cuando los vasos sanguíneos se constriñen, la circulación de sangre se torna lenta o se bloquea. El efecto leve o grave de la vasoconstricción, bien pudiera encontrarse relaciona con el uso de algunos medicamentos, pero también pudiera ser por el padecimiento de una enfermedad o al tipo de trastorno psicológico. (Enciclopedia Médica A.D.A.M., 2019)

Síntesis de prostaglandinas.

"La síntesis renal de las prostaglandinas es un mecanismo fisiológico para contrarrestar la disminución de la perfusión renal.” (García \& Gómez, 2000)

\section{Isquemia renal.}

“Reducción del flujo sanguíneo renal (hipoperfusión uni o bilateral) bien por disminución del volumen sanguíneo total, redistribución de la sangre u obstrucción." (Clínica Universidad de Navarra, 2019)

Perfusión renal.

Referido al flujo sanguíneo irrigado hacia a los riñones.

Sintomas de la IRA

En principio pudiera ser asintomática, pero si se llegan a presentar, pudieran ser todos o algunos de los siguientes problemas: 


\section{Injuria renal causada por AINEs}

Vol. 3, núm. 4., (2019)

Carolina Narcisa Chávez Ruíz; Luis Fernando Pascual García; Ximena Melissa Gonzabay Jiménez; Diana Lilibeth Cruz Yoza

- No orina lo suficiente

- Hinchazón o inflamación en las piernas, los tobillos o los pies

- Sensación de cansancio

- Problemas para recuperar el aliento

- Sentirse confundido

- Náuseas

- Dolor o presión en el pecho

- Puede tener convulsiones o caer en coma (en casos muy graves).

(American Kidney Fund, 2019)

Causas de la IRA

La ocurrencia generalmente es cuando repentinamente se dañan los riñones y puede ser debido a:

- No hay suficiente sangre fluyendo a través de sus riñones

- Una lesión directa a sus riñones o un problema con sus riñones

- Un bloqueo en sus uréteres, los tubos que llevan la orina de sus riñones a su vejiga

A su vez, la causa de la poca afluencia de sangre a través de sus riñones puede ser debido a alguno de los siguientes problemas:

- Presión arterial baja

- Sangrando excesivo

- Tener diarrea severa 


\section{Injuria renal causada por AINEs}

Vol. 3, núm. 4., (2019)

Carolina Narcisa Chávez Ruíz; Luis Fernando Pascual García; Ximena Melissa Gonzabay Jiménez; Diana Lilibeth Cruz Yoza

- Enfermedad cardíaca o ataque al corazón

- Infección

- Insuficiencia hepática

- El uso de AINEs.

- Quemaduras graves

- Estar muy deshidratado (no tener suficiente líquido en su cuerpo)

- Reacción alérgica severa

(American Kidney Fund, 2019)

De acuerdo con Serna \& Serrano (2012) se comprende que el compromiso renal es determinado de acuerdo al sitio de injuria, por lo tanto, éste se subdivide en: pre-renal, renal y post-renal.

En relación a lo anterior y al tema que aquí concierne, es decir, de la relación entre el uso de los AINEs y la determinación del compromiso renal asociado a su vez al sitio de injuria, es válido solo hacer referencias al: Compromiso Pre-Renal y Compromiso Renal. El primero de estos; asociado a la condición de disminución del flujo renal (causada principalmente por la depleción de volumen y la hipotensión), es porque el uso de los AINEs se encuentra igualmente vinculado como factor de riesgo, ya que éstos ocasionan un "deterioro de TFG por vasoconstricción en arteriola aferente". El segundo es porque, aparte de referirse a un origen vascular, glomerular o túbulo-intersticial, pudiera también deberse a la frecuente nefritis intersticial aguda, condición ésta que comúnmente asociada a medicamentos, estando entre esos AINEs. 


\section{Injuria renal causada por AINEs}

Vol. 3, núm. 4., (2019)

Carolina Narcisa Chávez Ruíz; Luis Fernando Pascual García; Ximena Melissa Gonzabay Jiménez; Diana Lilibeth Cruz Yoza

(Serna \& Serrano, 2012, págs. 173-174)

Calderón et al. (2011), en el mismo orden de ideas exponen que el daño renal inducido por los AINEs "se explica por la disminución en la concentración de las prostaglandinas", lo que derivará en una ineficiente función vasodilatadora a nivel de la arteriola aferente, un cambio en la presión intraglomerular y un detrimento en la tasa de filtración a este nivel.

Esta alteración de la hemodinámica intraglomerular puede conducir a una necrosis tubular aguda isquémica en la mayoría de los casos. Sin embargo, pueden describirse otras alteraciones histológicas como necrosis intersticial aguda, enfermedad de cambios mínimos, glomerulopatía membrano proliferativa, necrosis papilar aguda y esclerosis papilar. (Calderón et al.; 2011; p. 90)

Los expertos refieren que la relación que vincula el deterioro de la función renal y, por lo tanto, la nefrotoxicidad por la utilización de AINEs, se fundamenta más que todo en su amplio uso y automedicación, situación que a su vez se relaciona con el síndrome de abuso de analgésicos, inclusive.

Por su parte, Calvacanti et al.; (2018) indican que los riñones reciben aproximadamente el $25 \%$ de todo el gasto cardíaco de allí su importancia para la función excretora del cuerpo. Al activarse la síntesis de prostaglandinas como mecanismo regulador propio de la función de filtración, es sinónimo de una adecuada función de filtrado, lo que significa que mantendrá la tasa de filtración glomerular (TFG) y la homeostasis renal. 


\section{Injuria renal causada por AINEs}

Vol. 3, núm. 4., (2019)

Carolina Narcisa Chávez Ruíz; Luis Fernando Pascual García; Ximena Melissa Gonzabay Jiménez; Diana Lilibeth Cruz Yoza

En relación al efecto que ocasionan los AINEs sobre mencionada funcion renal, los mismos expertos continúan detallando que su uso:

[...] inhibe la cascada de ácido aracdónico, selectivamente o no, causando un efecto no permisivo sobre la formación de prostaglandinas. En los riñones, las prostaglandinas, principalmente prostaciclina, PGE2, PGD2, actuarán como vasodilatadores en las arteriolas aferentes, aumentando la perfusión renal, con distribución del flujo de la corteza a las nefronas en la región medular renal. Esta vasodilatación actúa como una contrarregulación de mecanismos como la acción del sistema renina-angiotensina-aldosterona y el sistema nervioso simpático, que culmina en una compensación para garantizar un flujo adecuado al órgano. El uso de AINEs inhibe este mecanismo y puede provocar vasoconstricción aguda e isquemia de la médula espinal, lo que puede provocar una lesión renal aguda.

Prosiguen los tratadistas alegando que la injuria (o lesión) renal aguda:

[...] comprende un síndrome caracterizado por una reducción abrupta de la TFG, que conduce a la retención de urea, creatinina y otras escorias nitrogenadas que normalmente se eliminan por los riñones. Esta condición se define clínicamente cuando los pacientes aumentan los niveles de creatinina en unos pocos días (o son 1,5 veces más altos que un resultado reciente o supuesto) o que desarrollan oliguria / anuria.

Las altas dosis de AINE han sido implicadas como causas de IRA, especialmente en los ancianos. Sin embargo, la AKI \{acrónimo ingles referido a Acute Kidnaey Injury; lo que en castellano es "IRA", por Injuria Renal Aguda\} mediada por AINE es una condición rara. [...] La 


\section{Injuria renal causada por AINEs}

Vol. 3, núm. 4., (2019)

Carolina Narcisa Chávez Ruíz; Luis Fernando Pascual García; Ximena Melissa Gonzabay Jiménez; Diana Lilibeth Cruz Yoza

forma principal de lesión renal aguda por AINE está mediada hemodinámicamente. Por el contrario, en situaciones de enfermedad renal crónica, insuficiencia cardíaca, insuficiencia hepática, shock hipovolémico y otras afecciones que reducen el volumen sanguíneo circulante, la secreción de estas hormonas aumenta para preservar la perfusión renal y la TFG. Romper este proceso con AINE conduce a una reducción de la perfusión renal intramedular y la isquemia, lo que aumenta el riesgo de necrosis tubular aguda (NTA). [...] La segunda presentación de AKI inducida por AINE es la nefritis intersticial aguda (NIA) con síndrome nefrótico. [...] Las dosis terapéuticas de AINE en pacientes susceptibles pueden causar daño renal agudo. La explicación de esto proviene del mismo mecanismo explicado anteriormente: la inhibición de la biosíntesis de prostanoides involucrados en el mantenimiento del flujo sanguíneo renal, específicamente PGE2 y PGI2. El riesgo es mayor en los recién nacidos y los ancianos, así como en pacientes con cualquier enfermedad cardiovascular, hepática, renal crónica o volumen sanguíneo circulante reducido, como los pacientes que usan AINE combinados con diuréticos e inhibidores del SARS. En un estudio de casos y controles de casi 80,000 usuarios a largo plazo de AINE asociados con diuréticos y / o inhibidores de la enzima convertidora de angiotensina (ECA), mostraron una relación firme al respecto. Se descubrió que la terapia combinada con monoterapia con AINE es responsable de un aumento absoluto en el riesgo de IRA adquirida en la comunidad en un año de uso, a pesar de la alta tasa de IRA causada por el uso exclusivo de medicamentos antiinflamatorios. siendo esta la clase mayoritaria en causar disfunción renal. (Cavalcanti et al.; 2018)

Bell, Rennie, Marwick \& Davey (2018) mediante su reciente investigación se enfocaron en analizar, principalmente, si los AINEs provocan que se eleven las tasas de IRA en pacientes con función renal normal sometidos a una cirugía; así como también, si dichos farmacos estan 


\section{Injuria renal causada por AINEs}

Vol. 3, núm. 4., (2019)

Carolina Narcisa Chávez Ruíz; Luis Fernando Pascual García; Ximena Melissa Gonzabay Jiménez; Diana Lilibeth Cruz Yoza

asociados con un mayor riesgo de muerte, y por último, si existen relación entre su uso y una mayor duración de la estancia hospitalaria y la necesidad de diálisis.

Estos investigadores, actualizando una revisión de ensayos controlados aleatorios (ECA) que compararon AINEs con placebo en pacientes con función renal normal sometidos a una cirugía, disponibles en el Registro Especializado Cochrane de Riñón y Trasplante, entre otras cosas hallaron que:

- En general, los AINES no tuvieron efectos claros sobre el riesgo de IRA posoperatoria, pueden aumentar ligeramente la creatinina sérica posoperatoria y no se puede precisar si dan lugar a la necesidad de terapia de reemplazo renal (TRR), provocan la muerte ni si aumentan la duración de la estancia hospitalaria.

- Los datos disponibles no confirman la seguridad de los AINES en los pacientes sometidos a una cirugía.

- Se necesitan estudios adicionales más grandes que utilicen la definición de IRA de los Kidney Disease Improving Global Outcomes, con pacientes con comorbilidades para confirmar estos resultados.

En otro importante estudio, Misurac et al. (2013), quienes tratando caracterizar la Lesión Renal Aguda (LRA) asociada a AINEs en los niños, mediante una revisión retrospectiva de gran magnitud de los pacientes de un hospital de niños de tercer nivel de atención, manejaron la hipótesis de que la depleción de volumen y/o la dosificación supraterapéutica de AINEs se asocia con mayor gravedad de la LRA asociada al AINEs. Sus resultados explicaron que los niños hospitalizados frecuentemente desarrollaban LRA, pero que comunmente era debido a múltiples 


\section{Injuria renal causada por AINEs}

Vol. 3, núm. 4., (2019)

Carolina Narcisa Chávez Ruíz; Luis Fernando Pascual García; Ximena Melissa Gonzabay Jiménez; Diana Lilibeth Cruz Yoza

causas. Indicaron que "Cuando un paciente sufre disminución de la perfusión renal secundaria a la disminución del volumen sanguíneo o a la disminución del volumen sanguíneo circulante eficaz, la perfusión renal se mantiene, en parte, por la generación intrarrenal de prostaciclina.”

Manifestaron que los AINEs inhiben las ciclooxigenasas I y II causando una disminución de la producción de prostaglandinas.

Así mismo, dijeron que hay poca evidencia de la disminución del flujo sanguíneo renal en el contexto del volumen circulante eficaz sea por el uso de AINEs, no obstante, al reducir el volumen circulante real o efectivo, éstos fármacos “disminuyen el flujo sanguíneo renal a través del bloqueo de la vasodilatación mediada por la prostaglandina en la arteriola preglomerular (aferente)", pudiendo en este particularidad ocasionar una retracción arteriolar preglomerular sin oposición mediante las acciones de las catecolaminas endógenas y otros compuestos vasoactivos. De la misma manera, ello pudiera conducir a una reducción de la tasa de filtración glomerular (TFG), baja de natriuresis, y en última instancia, como consecuencia del efecto combinado de los AINEs y la disminución del volumen efectivo circulante, hallana el camino hacia una isquemia renal y una necrosis tubular aguda (NTA). (Misurac et al.; 2013)

Con los resultados obtenidos en este estudio claramente se dió a enternder que, aún con dosis adecuadas, los niños que reciben AINEs estan sometidos a un alto riesgo de lesión renal aguda, y que, si bien es cierto que la evidencia marca un mayor riesgo en niños internados, no es menos cierto que en pacientes ambulatorios se plantea su causa/efecto. 


\section{Injuria renal causada por AINEs}

Vol. 3, núm. 4., (2019)

Carolina Narcisa Chávez Ruíz; Luis Fernando Pascual García; Ximena Melissa Gonzabay Jiménez; Diana Lilibeth Cruz Yoza

\section{Conclusión}

Tal y como se ha dejado evidencia en el transcurso del desarrollo de esta revisión, hoy más que antes se considera indispensable comprender que los AINEs, aunado muchas veces a su amplio e indiscriminado uso gracias a su fácil acceso, son un grupo de fármacos de los que, mediante varias clases de estudios y criterios científicos, se ha dejado evidencia que pueden producir múltiples afecciones a nivel renal.

A nivel clínico, es igualmente trascendente distinguir de manera adelantada a aquellos pacientes en los que se evidencie un particular riesgo elevado de ocurrencia de daño renal, habilidad que favorecerá en cuanto a la óptima prescripción y utilización de medicamentos, referidos a un mejor manejo de esquemas de dosificación, combinaciones posibles con otros fármacos y monitoreo, en pro de disminuir la influencia de este efecto adverso frecuente y potencialmente serio.

Es en base a todo lo antes referido que se vuelve imperioso siempre resaltar la importancia de que, tanto los profesionales de la salud como los pacientes, conozcan cada vez más a profundidad acerca de los efectos adversos que estos fármacos pueden causar a nivel renal, así como en otros órganos y sistemas.

\section{Referencias Bibliográficas}

American Kidney Fund. (2019). kidneyfund.org/en-espanol. Recuperado el 19 de 07 de 2019, de kidneyfund.org/en-espanol: http://www.kidneyfund.org/en-espanol/enfermedad-de-losrinones/otros-problemas-renales/lesion-renalaguda.html\#cuales_son_los_sintomas_de_una_lesion_aguda 


\section{Injuria renal causada por AINEs}

Vol. 3, núm. 4., (2019)

Carolina Narcisa Chávez Ruíz; Luis Fernando Pascual García; Ximena Melissa Gonzabay Jiménez; Diana Lilibeth Cruz Yoza

Bell, S. R., Marwick, C., \& Davey, P. (29 de Noviembre de 2018). cochrane.org/es. Recuperado el 18 de 07 de 2019, de cochrane.org/es: https://www.cochrane.org/es/CD011274/efectode-los-farmacos-antiinflamatorios-no-esteroideos-en-la-funcion-renal-de-pacientes-confuncion

Calderón, C., Guzmán, G., Sarmiento, J., Gómez, D., Joya, A., Ríos, L., . . . Soler, J. (2011). Nefrotoxicidad inducida por medicamentos. Maedicas Uis, 24(1), 65-85. Recuperado el 18 de $07 \quad$ de $2019, \quad$ de https://www.researchgate.net/publication/285787734_Nefrotoxicidad_inducida_por_med icamentos

Cavalcanti, G., Carneiro, A., Lima, R., Fagundes, M., Francesco, E., \& Bezerra, G. (21 de Septiembre de 2018). Aspectos fisiopatológicos de la nefropatía por fármacos antiinflamatorios no esteroideos. Revista Brasileña de Nefrología, 41(1). doi:10.1590/2175-8239-jbn-2018-0107

Clínica Universidad de Navarra. (2019). cun.es. Recuperado el 25 de 07 de 2019, de cun.es: https://www.cun.es/diccionario-medico/terminos/isquemia-renal

Enciclopedia Medica. (01 de Agosto de 2017). medlineplus.gov/spanish. Recuperado el 19 de 07 de 2019, de medlineplus.gov/spanish: https://medlineplus.gov/spanish/ency/article/000482.htm

Enciclopedia Médica A.D.A.M. (07 de Febrero de 2019). medlineplus.gov/spanish, Adaptada. (MedlinePlus, Editor) Recuperado el 25 de 07 de 2019, de medlineplus.gov/spanish: https://medlineplus.gov/spanish/ency/article/002338.htm

García, J., \& Gómez, J. (Enero de 2000). Fisiopatología de la ciclooxigenasa-1 y ciclooxigenasa2. Revista Española de Reumatología, 27(1), 33 - 35. Recuperado el 22 de 07 de 2019, de elsevier.es: https://www.elsevier.es/es-revista-revista-espanola-reumatologia-29-articulofisiopatologia-ciclooxigenasa-1-ciclooxigenasa-2-8546

Herrera, P. (19 de mayo de 2015). nefro.cl. Recuperado el 25 de 07 de 2019, de nefro.cl/: https://www.nefro.cl/v2/post.php?id=342

Hospital Aleman Asociación Civil. (2019). hospitalaleman.org.ar. Recuperado el 20 de 07 de 2019, de hospitalaleman.org.ar: https://www.hospitalaleman.org.ar/mujeres/injuria-renalaguda-una-enfermedad-reversible/

Kern Pharma. (09 de mayo de 2018). kernpharma.com/es/. Recuperado el 18 de 07 de 2019, de kernpharma.com/es/: https://www.kernpharma.com/es/blog/antiinflamatorio-o-analgesico 


\section{Injuria renal causada por AINEs}

Vol. 3, núm. 4., (2019)

Carolina Narcisa Chávez Ruíz; Luis Fernando Pascual García; Ximena Melissa Gonzabay Jiménez; Diana Lilibeth Cruz Yoza

Misurac, J., Knoderer, C., Leiser, J., Nailescu, C., Wilson, A., \& Andreoli, S. (Enero de 2013). Daño renal en niños. Antiinflamatorios no esteroides como causa de lesión renal aguda. The Journal of Pediatrics, 162(6), 1153 - 1159.e1. doi:doi.org/10.1016/j.jpeds.2012.11.069

Rechene, J., Fernández, P., \& Douthat, W. (2018). Fallo renal agudo en unidades críticas. Factores de riesgo y mortalidad. Rev Nefrol Dial Traspl. 2018; 38 (3): 170-8, 38(3), 170-178.

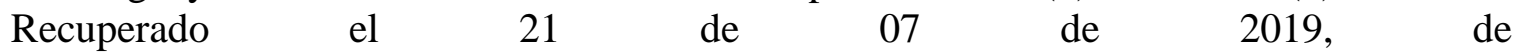
https://www.revistarenal.org.ar/index.php/rndt/article/view/353/356

Serna, J., \& Serrano, D. (2012). Injuria Renal Aguda. En C. Restrepo, C. Buitrago, J. Torres, \& J. Serna, Nefrología Básica 2 (2da. ed., págs. 169 - 177). Bogotá, Colombia: La Patria, S.A. Recuperado el 21 de 07 de 2019, de http://asocolnef.com/wpcontent/uploads/2018/03/Cap21.pdf

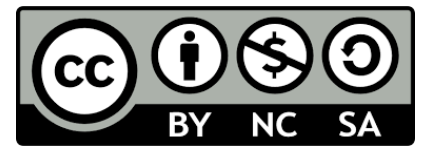

RECONOCIMIENTO-NOCOMERCIAL-COMPARTIRIGUAL

CC BY-NC-SA

ESTA LICENCIA PERMITE A OTROS ENTREMEZCLAR, AJUSTAR Y CONSTRUIR A PARTIR DE SU OBRA CON FINES NO COMERCIALES, SIEMPRE Y CUANDO LE RECONOZCAN LA AUTORÍA Y SUS NUEVAS CREACIONES ESTÉN BAJO UNA LICENCIA CON LOS MISMOS TÉRMINOS. 